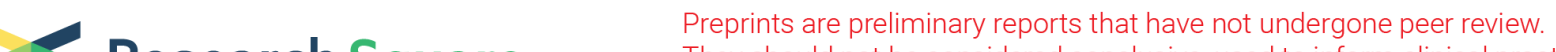 \\ They should not be considered conclusive, used to inform clinical practice, or referenced by the media as validated information.
}

\section{Understanding the Impacts Induced by Cut-Off Thresholds and Likelihood Measures on Confidence Interval When Applying GLUE Approach}

\section{Zhenya Li}

Hohai University https://orcid.org/0000-0002-5034-1677

Tao Yang ( $\nabla$ tao.yang@hhu.edu.cn )

Hohai University

Na Zhang

CWRC: Changjiang Water Resources Commission

\section{Yandong Zhang}

China water northeastern investigation

Jiahu Wang

Hohai University

Chongyu Xu

Univeristy of Oslo

\section{Pengfei Shi}

Hohai University

Youwei Qin

Hohai University

\section{Research Article}

Keywords: Generalized likelihood uncertainty estimate (GLUE) approach, confidence interval, likelihood measures, cut-off threshold, error model

Posted Date: April 30th, 2021

DOl: https://doi.org/10.21203/rs.3.rs-389101/v1

License: (c) (i) This work is licensed under a Creative Commons Attribution 4.0 International License.

Read Full License

Version of Record: A version of this preprint was published at Stochastic Environmental Research and Risk Assessment on February 9th, 2022. See the published version at https://doi.org/10.1007/s00477022-02182-6. 


\section{Abstract}

Generalized likelihood uncertainty estimate (GLUE) approach are heavily affected by the choices of cutoff thresholds and likelihood measures. This work attempts to study the potential mechanisms behind the impacts induced by cut-off thresholds and likelihood measures on confidence interval obtained by GLUE. A theoretical analysis on typical likelihood measures reveals that the error model of likelihood measure has essential impacts on the sampling processes of GLUE. Likelihood measures based on a same error model are mathematically transferrable, leading to an identical population of acceptable parameter sets. A case study is conducted by applying GLUE to uncertainty analysis on daily flows simulated by HBV model for the source region of the Yellow River basin. Seven interval indicators are adopted to describe the geometric features of confidence intervals, which are integrated into a comprehensive score for an overall assessment by multiple attribute decision making (MADM) framework. Results indicate that 1) With an increase of cut-off threshold, confidence interval widens in low-level flow sections, moves upward in recession phases of medium-level flow sections whereas narrows in high-level flow sections. Trade-off mechanism amongst widening, moving and narrowing trends is a potential reason behind the variations of interval indicators with cut-off threshold. 2) Much higher similarities in confidence intervals can be detected for likelihood measures based on a same error model than those based on different error models; 3 ) increasing cut-off threshold highlights the impacts induced by the error models of likelihood measures, whereas weakens the impacts induced by the formulas of likelihood measures.

\section{Full Text}

This preprint is available for download as a PDF.

\section{Figures}




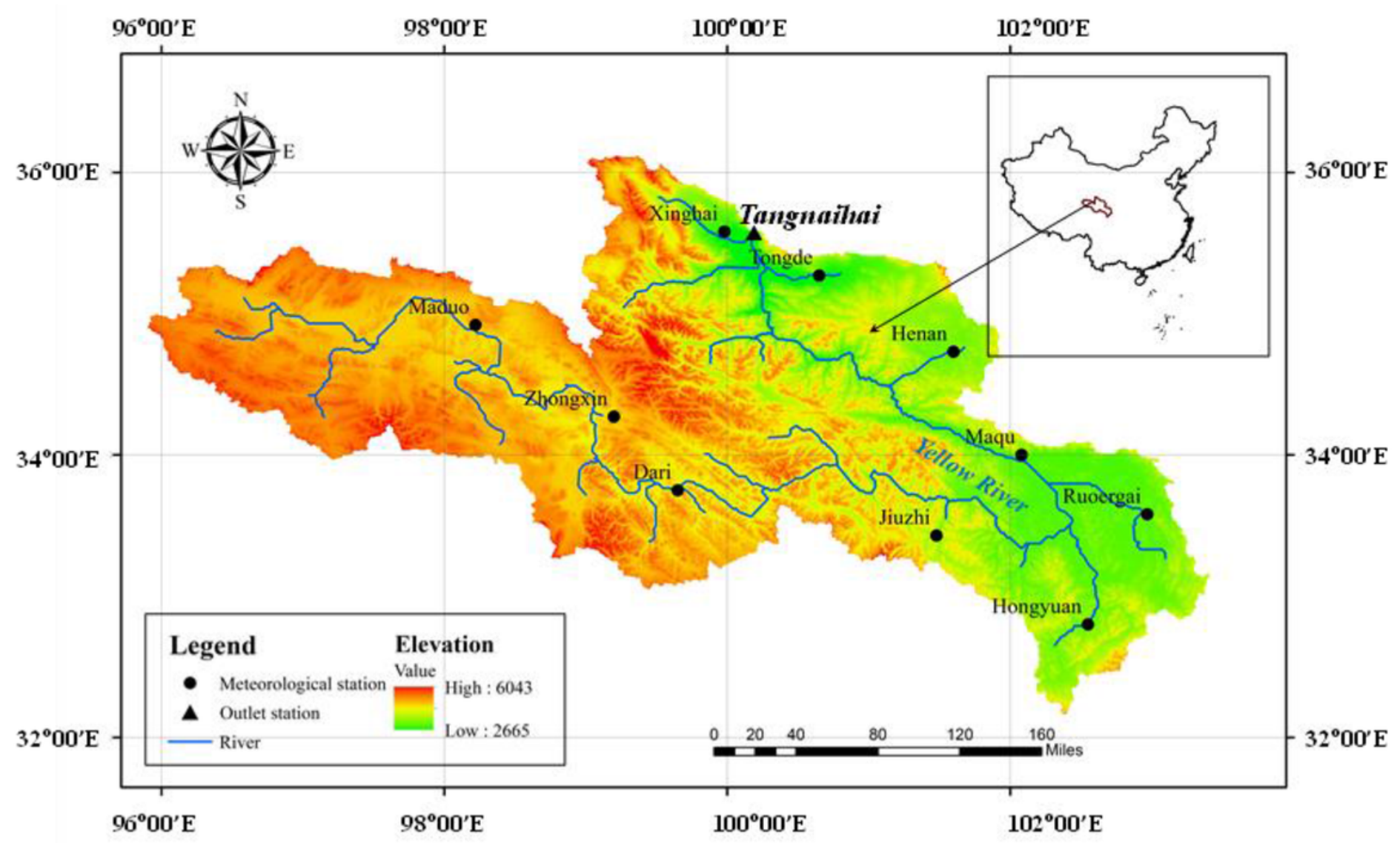

Figure 1

Map of the source region of the Yellow River basin Note: The designations employed and the presentation of the material on this map do not imply the expression of any opinion whatsoever on the part of Research Square concerning the legal status of any country, territory, city or area or of its authorities, or concerning the delimitation of its frontiers or boundaries. This map has been provided by the authors.
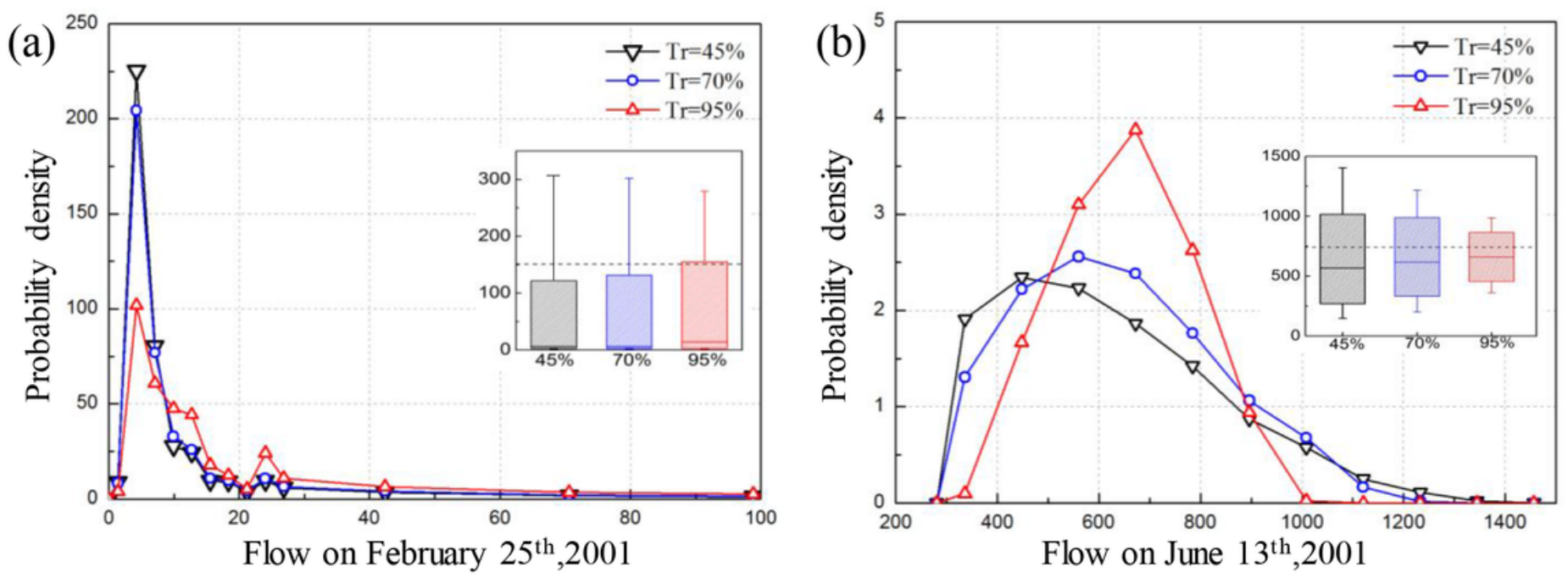

Figure 2 
PPD distributions and box plots of model outputs on February 25th, 2001 and June 13rd, 2001 at Tr=45, 70 and $95 \%$. Note: the box plots show the 0th percentile (i.e. lower cap), 5 th percentile (i.e. bottom of the box), 50th percentile (i.e. line in the box), 95th percentile (i.e. top of the box) and 100th percentile (i.e. upper cap) of model outputs together with observed flow (i.e. dashed line)

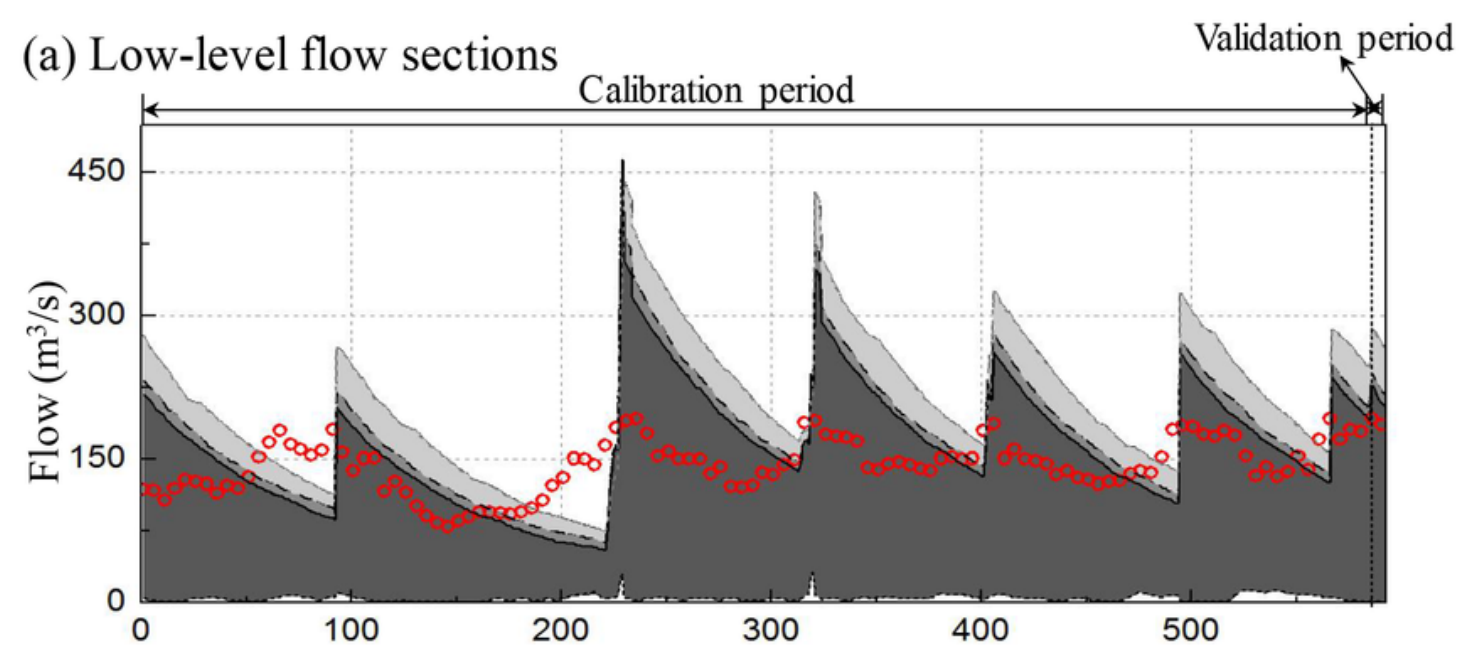

(b) Medium-level flow sections

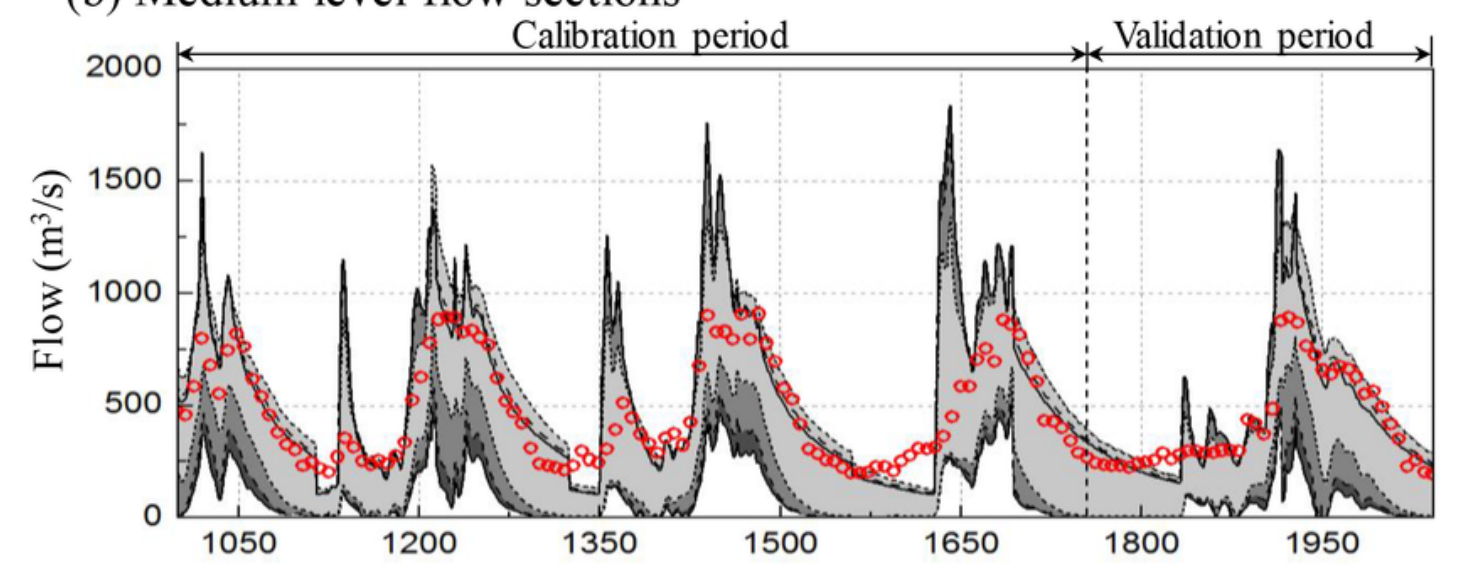

(c) High-level flow sections

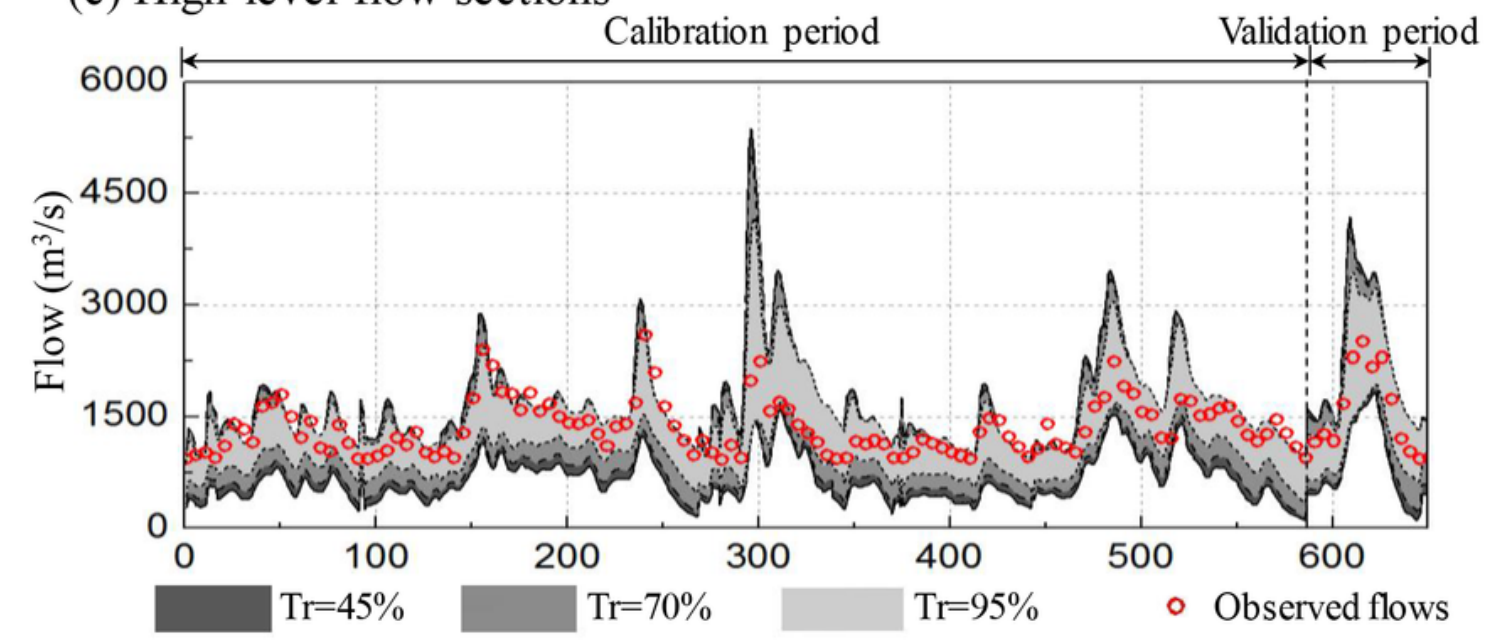

Figure 3 
90\% GLUE intervals of (a) low-level (b) medium-level and (c) high-level flow sections over calibration and validation periods
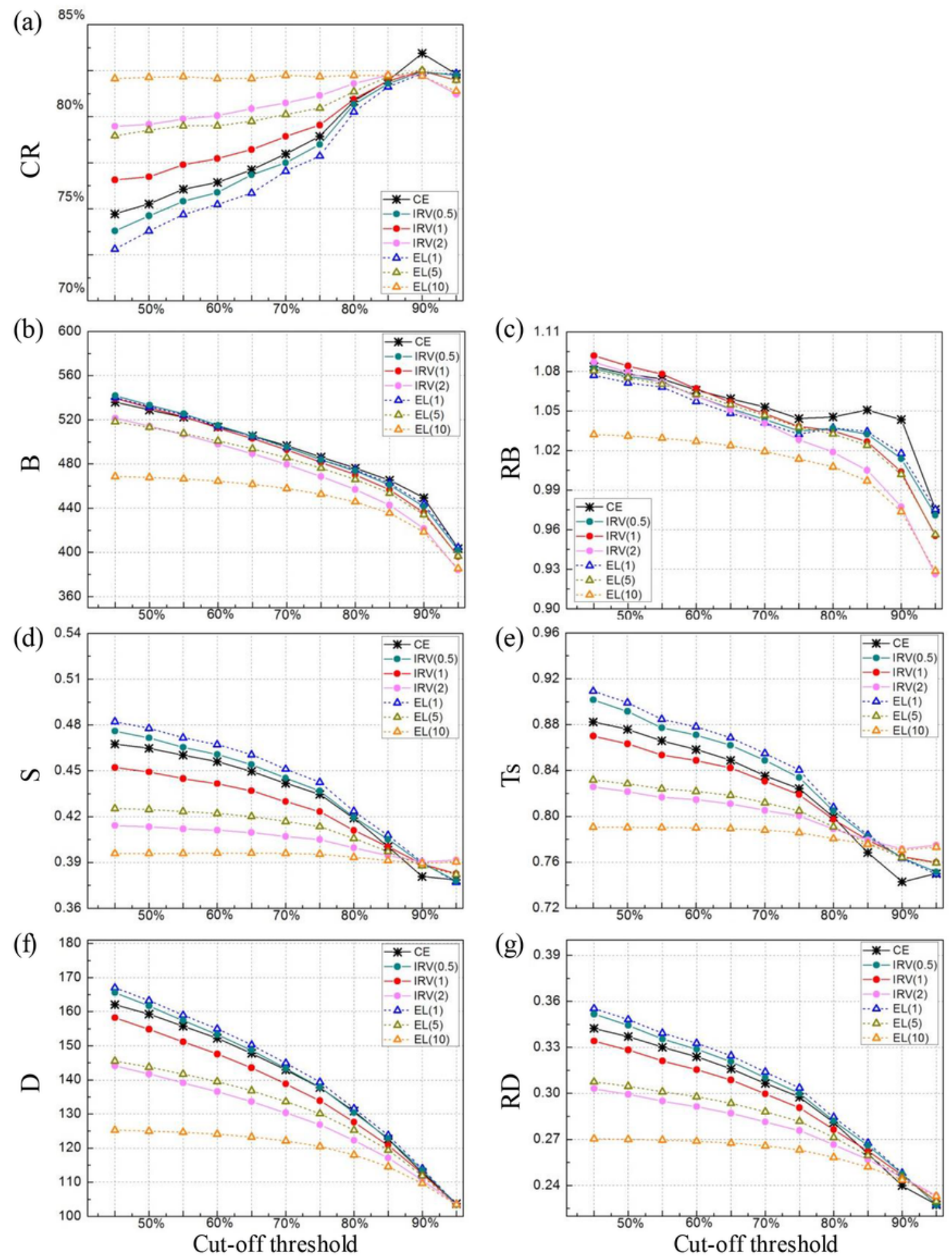

\section{Figure 4}

Interval indicators of $90 \%$ GLUE intervals obtained by likelihood measures based on a same error model in calibration period 

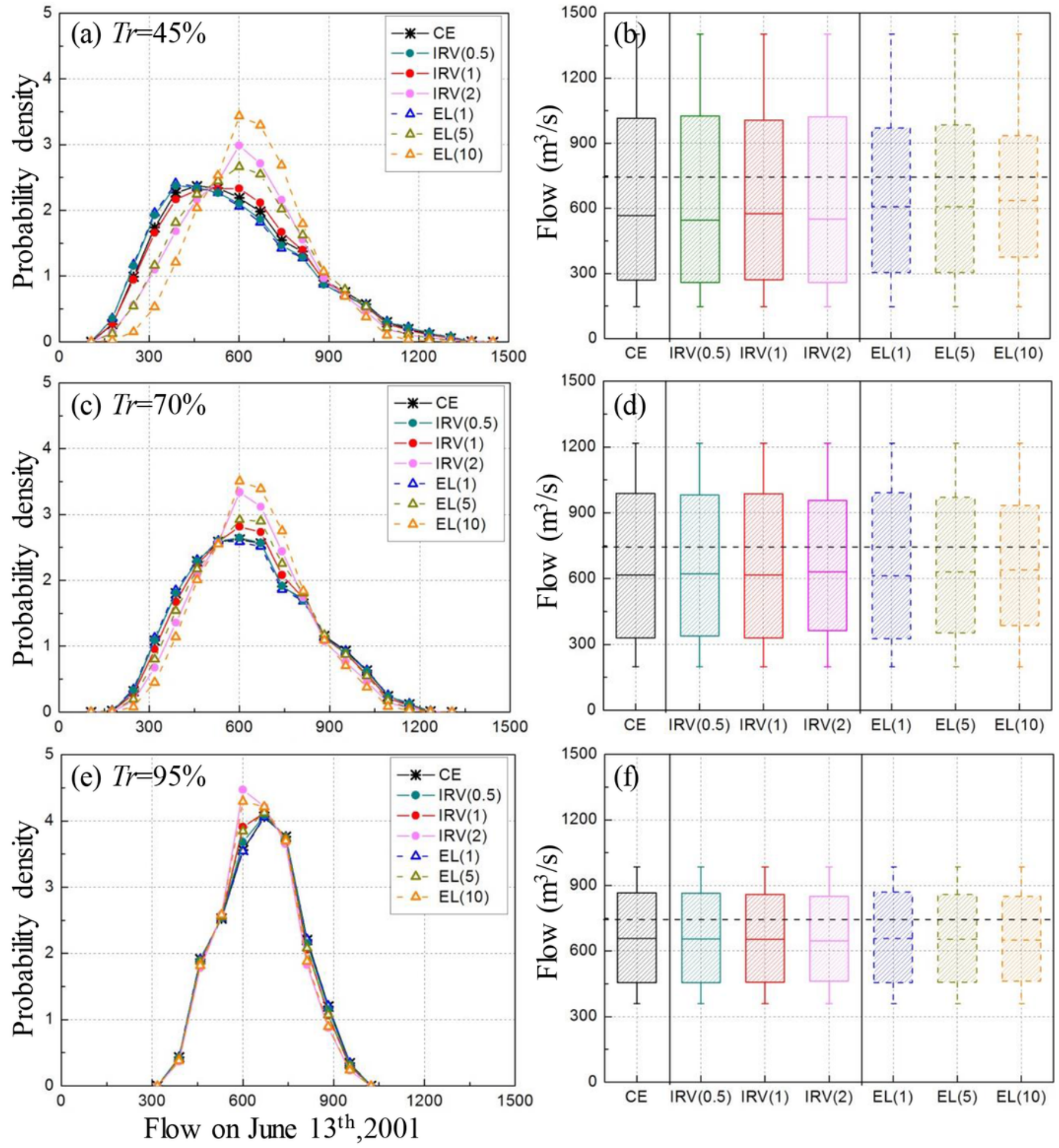

\section{Figure 5}

PPD distributions and box plots of model outputs on June 13rd, 2001 obtained by likelihood measures based on a same error model 

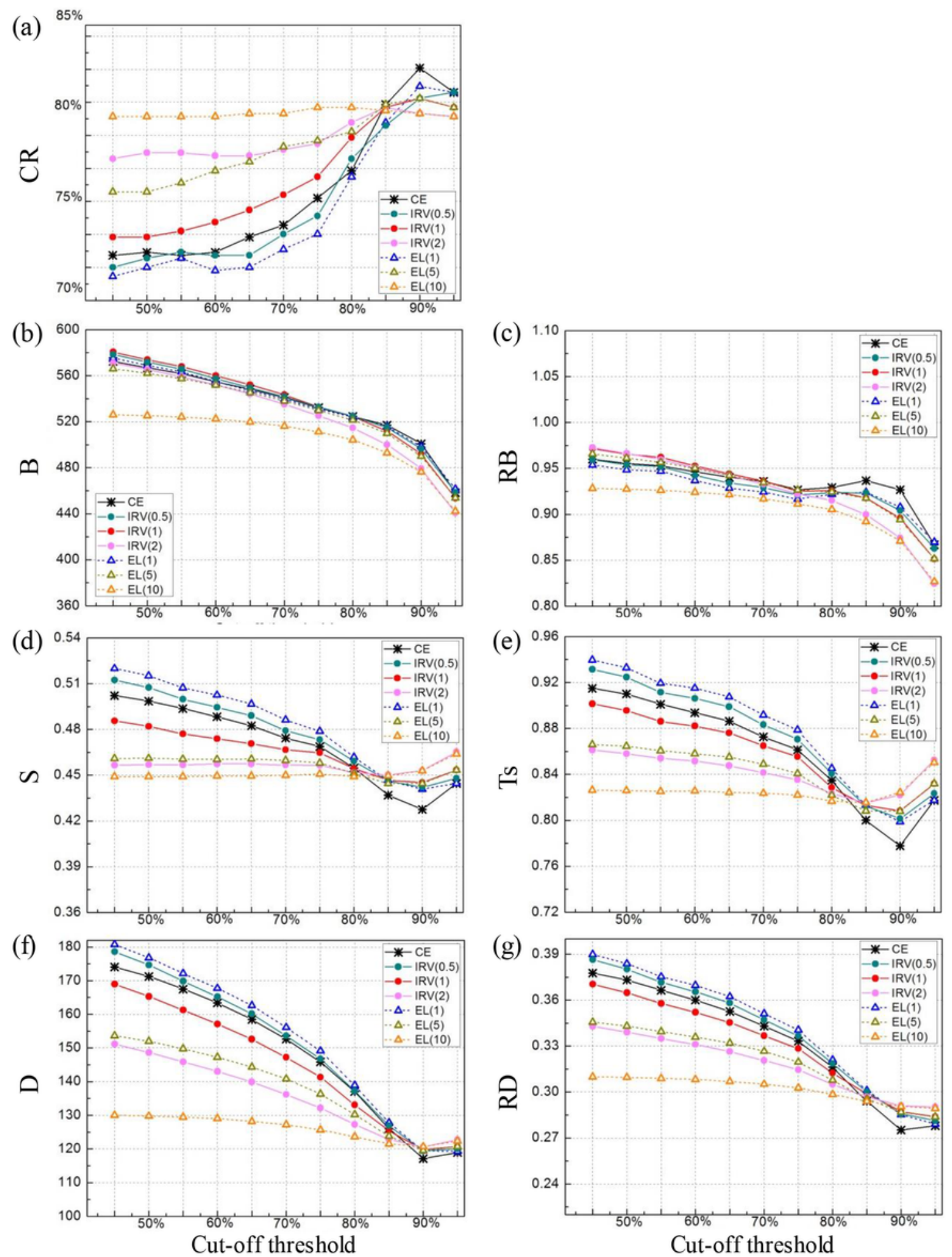

\section{Figure 6}

Interval indicators of $90 \%$ GLUE intervals obtained by likelihood measures based on a same error model in validation period 
(a) Calibration period

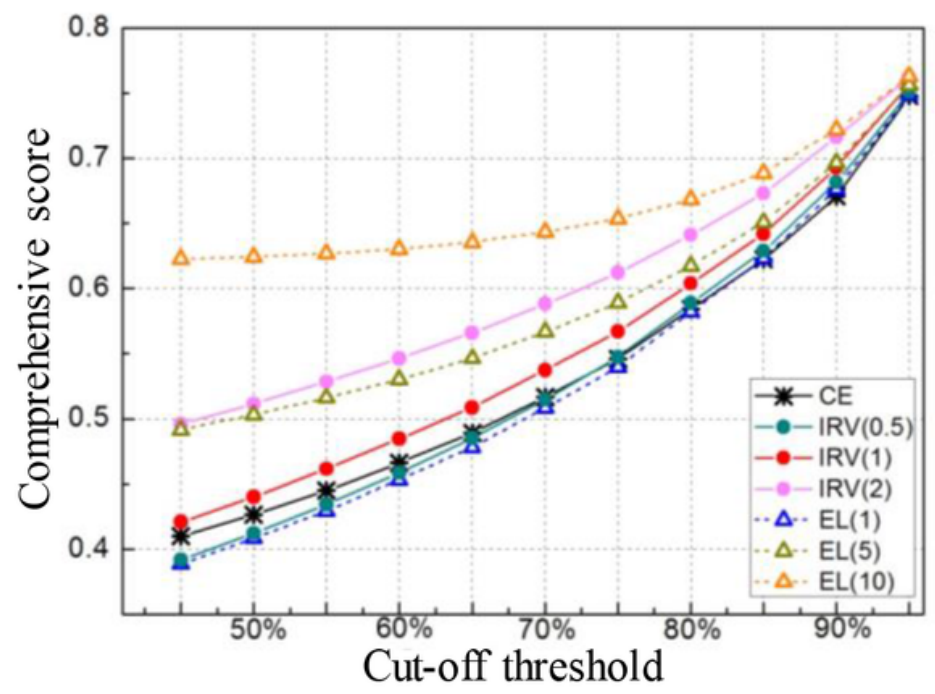

(b) Validation period

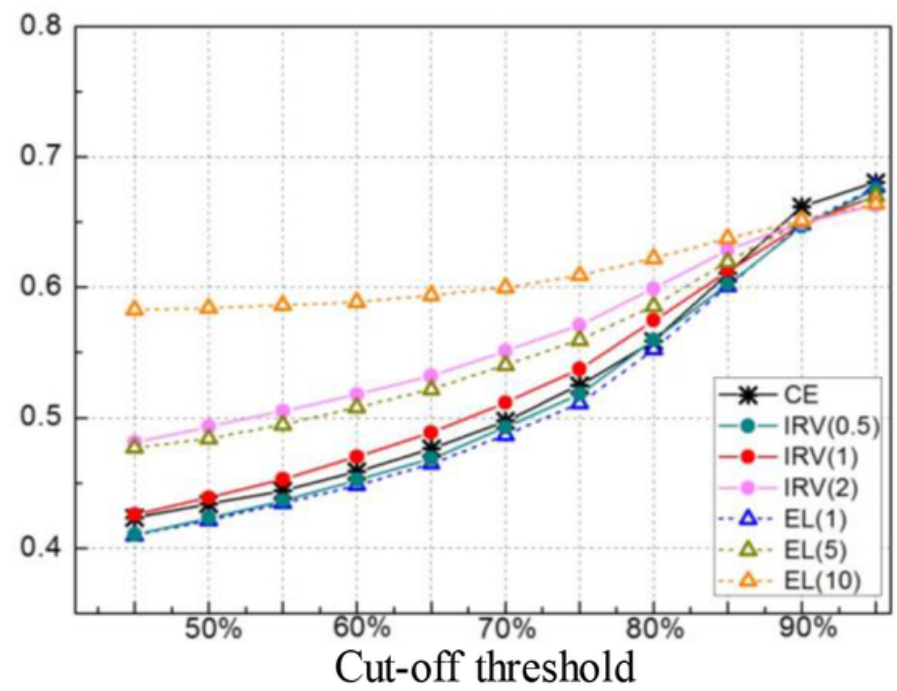

Figure 7

Comprehensive scores (CSs) of $90 \%$ GLUE intervals by likelihood measures based on a same error model 

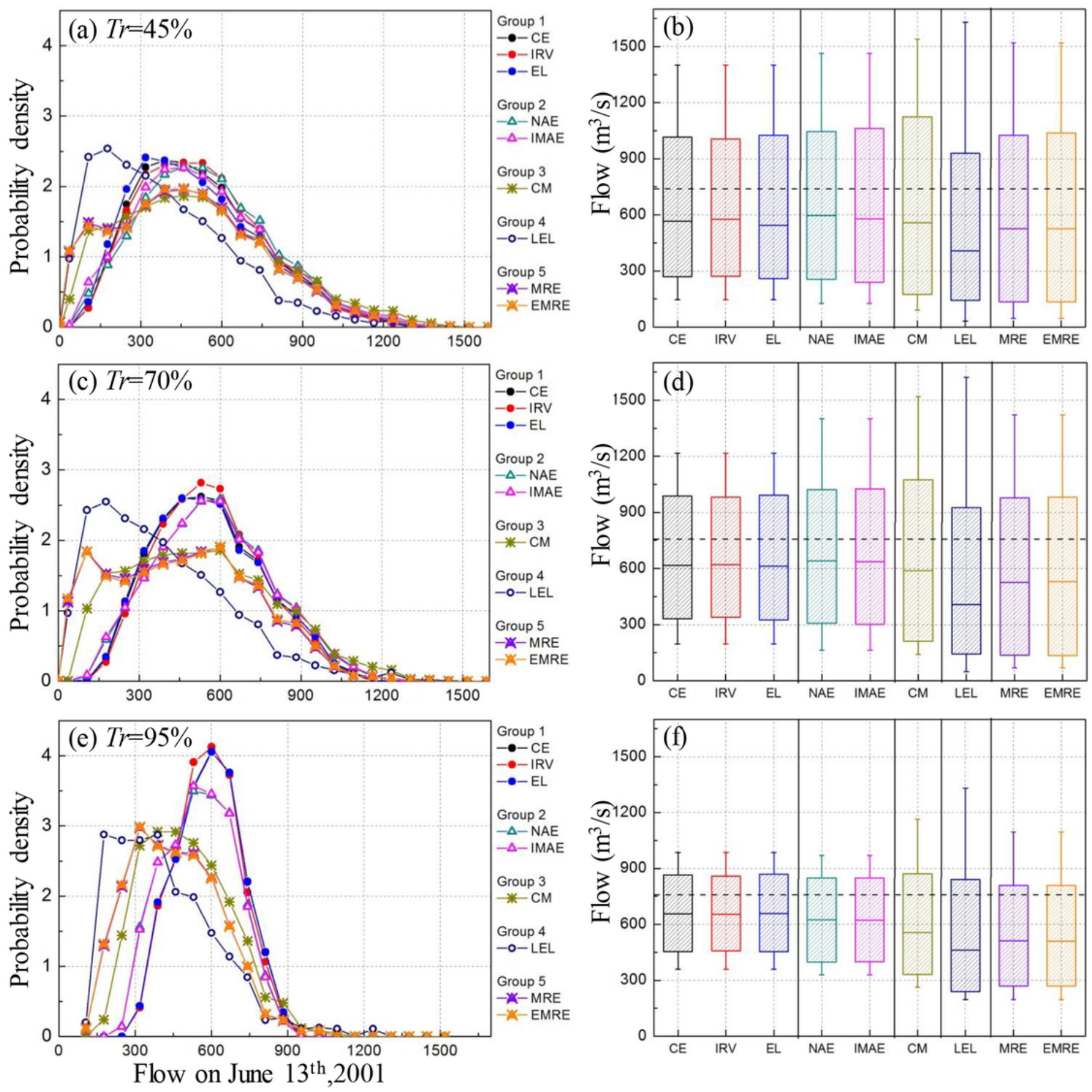

Figure 8

PPD distributions and box plots of model outputs on June 13rd, 2001 obtained by likelihood measures based on different error models 

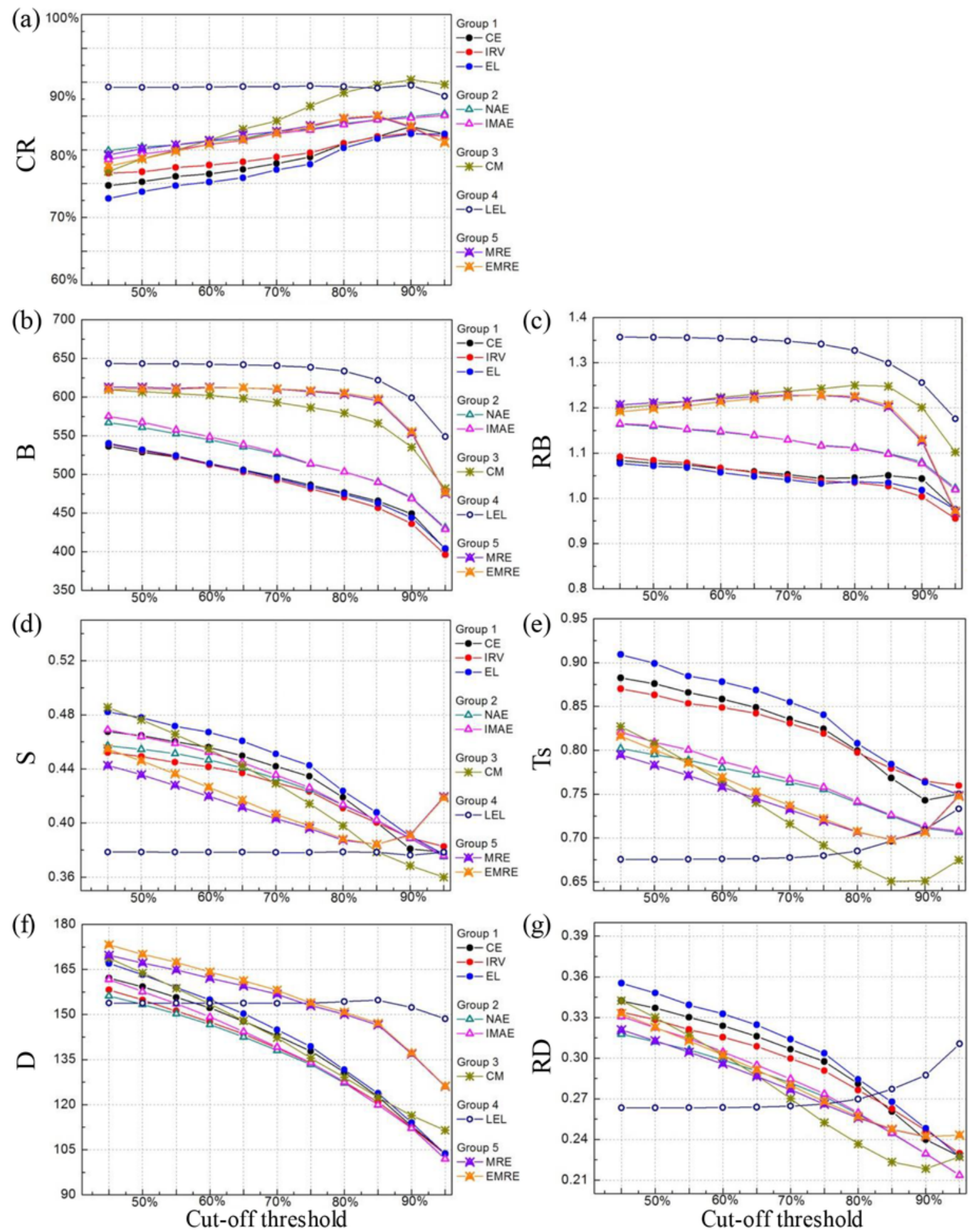

\section{Figure 9}

Interval indicators of $90 \%$ GLUE intervals obtained by likelihood measures based on different error models in calibration period 

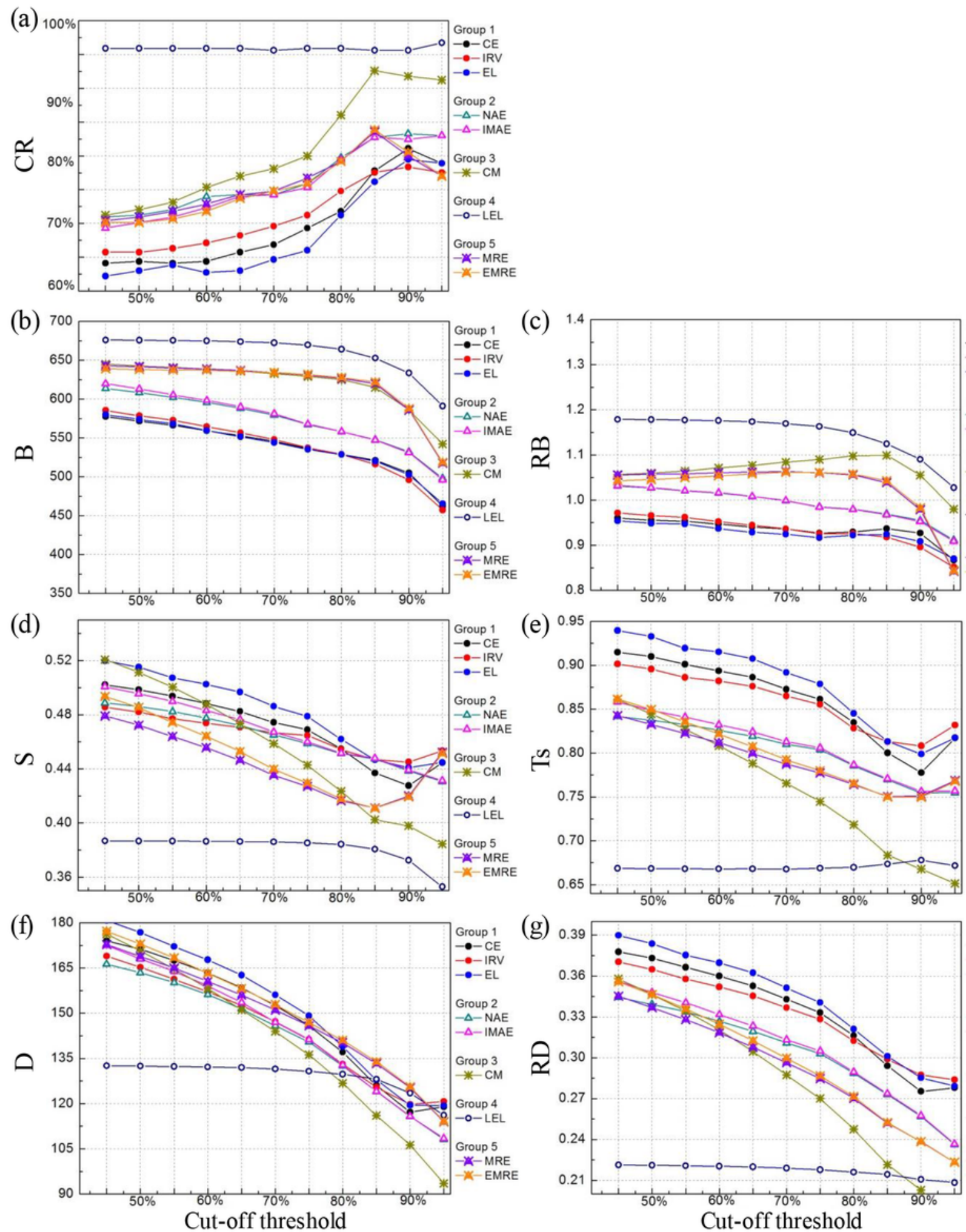

Figure 10

Interval indicators of $90 \%$ GLUE intervals obtained by likelihood measures based on different error models in validation period 
(a) Calibration period

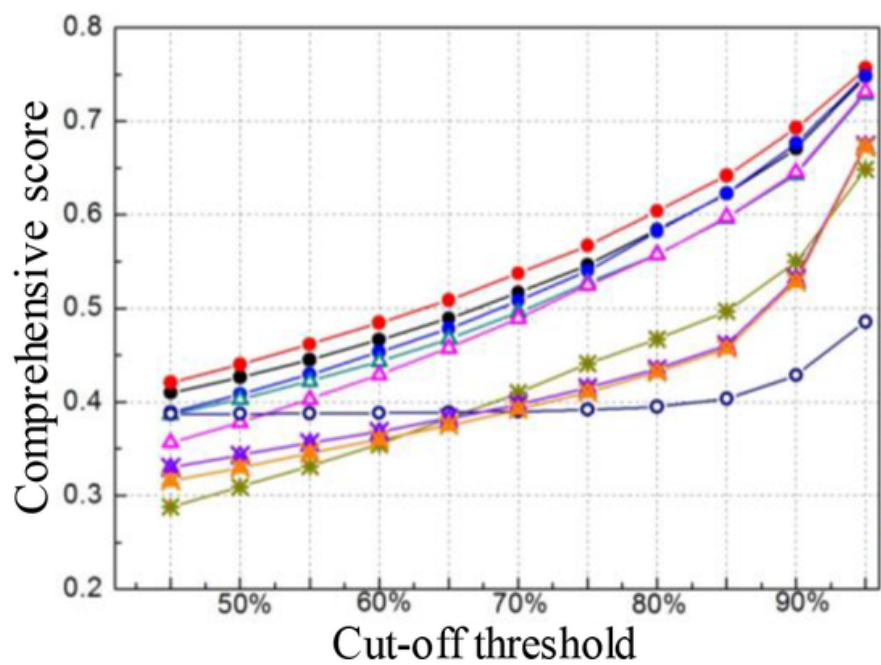

(b) Validation period

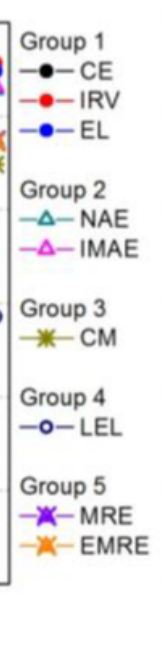

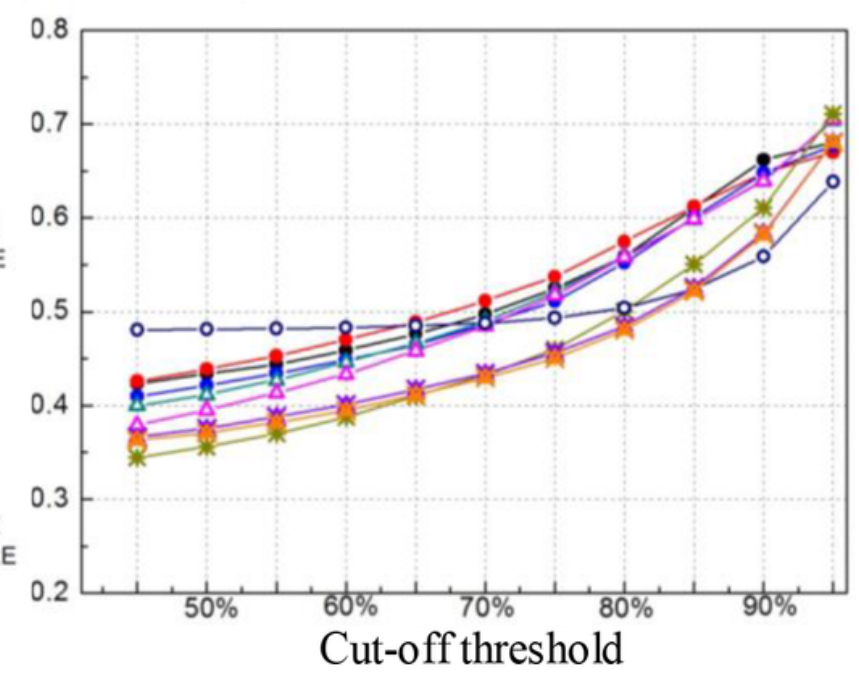

Figure 11

Comprehensive scores (CSs) of $90 \%$ GLUE intervals by likelihood measures based on different error models 